\title{
O manejo da epilepsia na atenção primária à saúde e o que mudou com a pandemia da COVID-19?
}

\section{Epilepsy management at the primary care setting and what has changed with the COVID-19 pandemic?}

Júlia Castelan Bastian', Gigliolle Romancini de Souza Lin ${ }^{2}$, Jaime Lin ${ }^{3}$, Mariana dos Santos Lunardi', Katia Lin ${ }^{5}$

1. Médica. Residente em Oftalmologia, Hospital das Clínicas da Faculdade de Medicina da Universidade de São Paulo (HCFMUSP). São Paulo, SP, Brasil.

2. Médica da Família e Comunidade. Professora do Curso de Graduação em Medicina da Universidade do Sul de Santa Catarina (UNISUL). Tubarão, Santa Catarina, Brasil.

3. Médico neuropediatra. Mestre em Neurologia. Professor do Curso de Graduação em Medicina da Universidade do Sul de Santa Catarina (UNISUL). Tubarão, Santa Catarina, Brasil.

4. Médica neurologista, Doutora em Ciências Médicas pelo Programa de Pós-Graduação em Ciências Médicas da Universidade Federal de Santa Catarina. Hospital Governador Celso Ramos. Florianópolis, Santa Catarina, Brasil.

5. Médica Neurologista. Doutora em Neurologia. Professora do Departamento de Clínica Médica e do Programa de Pós-graduação em Ciências Médicas, Centro de Ciências da Saúde, Universidade Federal de Santa Catarina, Campus Prof. João David Ferreira Lima. Florianópolis, SC, Brasil.

\section{RESUMO}

A epilepsia é uma doença neurológica bastante comum, acometendo aproximadamente 4 milhões de pessoas no Brasil e com uma estimativa de 150.000 novos casos diagnosticados todos os anos. Uma condição tão prevalente não poderia ser manejada apenas por especialistas focais, sendo imprescindível que a Atenção Primária à Saúde (APS) exerça um papel forte e resolutivo, ordenando as redes de atenção à saúde. Neste contexto, é de fundamental importância o papel do Médico de Família e Comunidade (MFC) no diagnóstico e tratamento da condição, bem como a participação da APS, desempenhando maior articulação com os diferentes pontos de atenção, a fim de garantir a integralidade do cuidado da pessoa com epilepsia.

\section{ABSTRACT}

Epilepsy is a common neurological disease, affecting approximately 4 million people in Brazil and with an estimated 150,000 new cases diagnosed each year. Focal specialists alone should not manage such a prevalent condition. It is essential that Primary Health Care (PHC) plays a strong role and rationally orders health care networks. In this context, it is of fundamental importance to instruct the Family and Community Doctors to properly diagnose and treat this condition. Additionally, it is vital that PHC articulates different points of care, in order to guarantee the integral care of the person with epilepsy.

DOI: https://doi.org/10.32963/bcmufsc.v7i1.4532

Indexadores: Epilepsia; Atenção primária em saúde; Medicamentos antiepilépticos; Epilepsia farmacorresistente.

Keywords: Epilepsy; Primary health care; Anticonvulsants; Drug-resistant epilepsy Submetido em 13/12/2020; aceito para publicação em 3/4/2021.

Os autores não possuem conflitos de interesse referentes ao presente trabalho.

Apoio: CNPq (Conselho Nacional de Desenvolvimento Científico e Tecnológico) e FAPESC (Fundação de Amparo à Pesquisa e

Inovação do Estado de Santa Catarina)

Autor para contato: Profa. Katia Lin. E-mail: katia.lin@ufsc.br

\section{Introdução}

As epilepsias são um grupo de doenças neurológicas caracterizadas por uma atividade elétrica exagerada do parênquima cerebral e por uma predisposição à ocorrência de crises epilépticas espontâneas. A definição mais atual de "epilepsia" foi proposta pela Liga Internacional Contra as Epilepsias (ILAE) como: "desordem do cérebro caracterizada pela predisposição a gerar crises epilépticas e pelas consequências neurobiológicas, cognitivas e sociais desta condição". A ILAE também define o termo "crise epiléptica" como a "ocorrência transitória de um sinal ou 
sintoma decorrente de uma atividade neuronal anormal, cxcessiva e síncrona do cérebro ${ }^{m, 2}$.

Estima-se uma prevalência mundial de epilepsia entre $0,5 \%$ a $1,0 \%$ da população, o que se traduz em um número absoluto de aproximadamente 65 milhões de pessoas. No Brasil, a prevalência é de $1,8 \%$, equivalendo a um total de 4 milhões de brasileiros e 150.000 casos novos diagnosticados a cada ano ${ }^{3,4}$.

Esses pacientes enfrentam crises limitadoras de suas atividades diárias, comprometimento neurocognitivo, comorbidades psiquiátricas e dependência social. $\mathrm{O}$ tratamento inadequado ou ausente, pode atingir $80 \%$ do total de pacientes no Brasil, sendo imprescindível, portanto, o papel da atenção primária em saúde (APS) para ampliar o acesso e melhorar o cuidado dessa condição ${ }^{5,6}$.

A atual pandemia da COVID-19 iniciada no final do ano de 2019 impôs mudanças profundas em todo o globo, não somente nos hábitos de vida, mas também no cuidado à saúde. Iniciativas envolvendo a telemedicina no tratamento da epilepsia precederam a pandemia em vários países, como Canadá, Argentina, zonas rurais dos Estados Unidos, em regiões desassistidas do pólo norte, e no Paquistão desde 2008. No tratamento da epilepsia, em particular, maioria das consultas de seguimento (follow-up visits) necessitam apenas de uma conversa para avaliação da frequência atual de crises, uso e adesão ao tratamento medicamentoso, rastreamento de efeitos adversos e comorbidades psiquiátricas, com muitas vantagens para o paciente, que pode ser consultado em seu ambiente familiar onde pode agir mais naturalmente, economiza tempo, dinheiro e os riscos de ter que deixar sua moradia (risco de ter crises epilépticas, acidentes, violência urbana, contágio da COVID-19, entre outros), bem como permite educação em saúde de acompanhantes e familiares e consultas multidisciplinares sem necessidade de deslocamento ${ }^{7.8}$.

O presente artigo discute, portanto, os cuidados da epilepsia, com enfoque específico no paciente adulto, sendo organizado nas seguintes seções: (1) Avaliação do paciente com epilepsia (PCE) na APS; (2) Exames complementares e diagnóstico; (3) Abordagem terapêutica inicial; (4) Epilepsia farmacorresistente e quando encaminhar para o especialista; (5) Riscos do PCE frente a pandemia da COVID-19.

\section{Métodos}

Trata-se de uma revisão narrativa e nãosistemática, de leitura objetiva e pautada em evidências que busca sintetizar o manejo da epilepsia na APS, aliando-se à experiência clínica dos autores, os quais implantaram o programa de atendimento ao paciente com epilepsia de Santa Catarina, Brasil. Para tanto, realizou-se uma revisão da literatura na base de dados PubMed/Medline, SCOPUS e LILACS, utilizando-se as expressões "epilepsia", "epilepsia recém-diagnosticada", "primeira crise não provocada", "epilepsia resistente a medicamentos", "atenção primária à saúde", e "anticonvulsivantes". Foram selecionados artigos originais e de revisão, publicados na língua inglesa e portuguesa, sem restrição de data e com data final de busca em 30/novembro/2020.

\section{Avaliação do paciente com crises epilépticas na APS}

As crises epilépticas expressam-se por uma ampla variedade de manifestações, a depender da origem do ictus, da extensão do acometimento cerebral e da presença de comorbidades associadas. O diagnóstico da epilepsia é clínico e o primeiro objetivo é determinar se realmente a crise é epiléptica, classificála e buscar indícios de que esta recorrerá. O Quadro 1 apresenta os critérios clínicos definidos pela ILAE para o diagnóstico ambulatorial de epilepsia'.

Em seguida, deve-se proceder à diferenciação das crises epilépticas e de seus diagnósticos diferenciais (Quadro 2). Xu et al10 revela que aproximadamente $25 \%$ dos pacientes são diagnosticados erroneamente com epilepsia e na realidade, apresentam eventos nãoepilépticos. No diagnóstico diferencial, a investigação da história clínica é fundamental, devendo-se questionar a semiologia da crise, idade de início, frequência, fatores desencadeantes, ocorrência de perda de consciência, presença de aura associada e outras comorbidades. $\mathrm{O}$ relato dos acompanhantes que presenciaram a crise também é um grande aliado para elevar a acurácia diagnóstica.

$O$ exame físico com ênfase nas avaliações neurológica e psiquiátrica, pode complementar com indícios valiosos a etiologia das crises. O grande desafio consiste em que, frequentemente, na epilepsia, o exame físico é normal, pois durante o período interictal, não há alterações neurológicas perceptíveis. Já nas situações em que uma condição de base esteja acarretando as crises, o exame físico pode indicar as etiologias mais prováveis? 
Quadro 1. Critérios operacionais da Liga Internacional Contra as Epilepsias (ILAE) para o diagnóstico de epilepsia.

1. Ao menos duas crises epilépticas não-provocadas (ou reflexas) com intervalo $>24 \mathrm{~h}$

2. Uma crise não provocada com probabilidade de ocorrência de novas crises semelhante à probabilidade de recorrência após duas crises não provocadas (ao menos 60\%)

3. Diagnóstico clínico de uma síndrome epiléptica

Fonte: Adaptado de Fisher et al, $2014^{1}$.

Quadro 2. Diagnósticos diferenciais das crises epilépticas e sua prevalência.

\begin{tabular}{|l|l|}
\hline Causas & Principais exemplos \\
\hline Síncopes $(52,4 \%)$ & Cardiogênicas, vasovagais \\
\hline Psicogênicas (34,7\%) & Transtorno conversivo, crises de pânico \\
\hline Neuropsiquiáticas (3,2\%) & Transtorno do espectro autista, deficiência cognitiva, distúrbios do aprendizado \\
\hline $\begin{array}{l}\text { Distúrbios do movimento } \\
\text { Cefaleias (2,2\%) }\end{array}$ & $\begin{array}{l}\text { Coreia, balismo, discinesias paroxísticas, distonia, mioclonia, hemiplegia, } \\
\text { tremores, coreoatetose }\end{array}$ \\
\hline $\begin{array}{l}\text { Distúrbios do sono (1,3\%) } \\
\text { Quadros confusionais agudos }\end{array}$ & Delirium, quadros demenciais, confusão mental pós-trauma \\
\hline $\begin{array}{l}\text { Cerebrovasculares }(0,7 \%) \\
\text { e amnésicos }(0,8 \%)\end{array}$ & Acidente vascular encefálico, acidente isquêmico transitório com aura \\
\hline
\end{tabular}

Fonte: Adaptado de Xu et al, $2016^{10}$.

Após descartados os diagnósticos diferenciais, deve-se partir para a classificação das crises. A classificação mais atual das epilepsias foi elaborada pela Comissão de Classificação e Terminologia da ILAE, em 2017 (Quadro 3) e propõe que a determinação do tipo de crise, tipo de epilepsia e a síndrome epiléptica seja feita na abordagem inicial da pessoa com epilepsia ${ }^{2}$. Segundo este consenso, as crises devem ser divididas em focais, generalizadas ou combinadas. Nas crises focais, a origem é localizada e suas manifestações clínicas dependem do local de início e da extensão com que se propaga a descarga epiléptica. Este é o tipo mais frequente e acomete $70 \%$ dos adultos com epilepsia recentemente diagnosticada ${ }^{10}$.

Já as crises generalizadas comprometem ambos os hemisférios cerebrais, em redes neuronais de distribuição bilateral. Esta classe geralmente resulta em alteração do nível de consciência, sendo dividida em crises motoras e não-motoras. Eventualmente, os estímulos focais podem propagar-se para todo o córtex cerebral, resultando em uma crise focal evoluindo para tônico-clônica bilateral'. 
Quadro 3. Classificação vigente das crises epilépticas segundo a Liga Internacional Contra as Epilepsias (ILAE).

\begin{tabular}{|c|c|c|c|}
\hline \multicolumn{2}{|l|}{ Início focal } & Início generalizado & Início desconhecido \\
\hline Perceptiva & Disperceptiva & Motor & Motor \\
\hline \multicolumn{2}{|c|}{$\begin{array}{l}\text { Início motor: } \\
\text { - Automatismos } \\
\text { - Atônicas } \\
\text { - Clônicas } \\
\text { - Espasmos epilépticos } \\
\text { - Hipercinéticas } \\
\text { - Mioclônicas } \\
\text { - Tônicas }\end{array}$} & $\begin{array}{ll}\text { - } & \text { Tônico-clônicas } \\
\text { - } & \text { Clônicas } \\
\text { - } & \text { Mioclônicas } \\
\text { - } & \text { Mioclono-tônico-clônicas } \\
\text { - } & \text { Mioclono-atônicas } \\
\text { - Atônicas } \\
\text { - Espasmos epilépticos } \\
\text { Não-motor (ausência): } \\
\text { - Típicas } \\
\text { - } \quad \text { Mtípicas } \\
\text { - Mioclônicas } \\
\text { Mioclonias palpebrais }\end{array}$ & $\begin{array}{l}\text { • Tônico-clônicas } \\
\text { • Espasmos epilépticos } \\
\text { Não-motor: } \\
\text { • Parada comportamental }\end{array}$ \\
\hline $\begin{array}{l}\text { Focal evol } \\
\text { bilateral }\end{array}$ & ara tônico-clônica & & Não classificadas \\
\hline
\end{tabular}

Fonte: Fisher et al, $2017^{2}$.

A ILAE também ressalta a importância de se determinar a etiologia das crises, pelas implicações diretas na terapêutica e prognóstico. As principais etiologias causadoras das epilepsias são, primeiramente, as alterações estruturais, seguidas das genéticas, metabólicas, imunológicas e infecciosas² (Quadro 4). Nesta investigação é fundamental questionar sobre antecedentes de complicações pré-natais e perinatais, bem como traumas, intoxicações e infecções neurológicas prévias.

\section{Quadro 4. Principais etiologias das epilepsias.}

\begin{tabular}{|l|l|}
\hline Etiologia & Principais causas \\
\hline Estrutural & $\begin{array}{l}\text { Lesões isquêmicas, trauma, neoplasias cerebrais, anomalias vasculares, malformações do } \\
\text { desenvolvimento cortical, esclerose mesial temporal }\end{array}$ \\
\hline Genética & EMJ, ELF, EAI, SD \\
\hline Infecciosa & $\begin{array}{l}\text { Neurocisticercose, neurotuberculose, neuroinfecção por HIV, infecção congênita por } \\
\text { citomegalovírus, meningites }\end{array}$ \\
\hline Metabólica & \begin{tabular}{l} 
Uremia, porfiria, aminoacidopatias \\
\hline Imunológica
\end{tabular} \\
$\begin{array}{l}\text { Encefalite límbica autoimune; Encefalopatia de Hashimoto; Encefalite autoimune anti- } \\
\text { NMDA }\end{array}$ \\
\hline
\end{tabular}

Siglas: EMJ = Epilepsia mioclônica juvenil; ELF = Epilepsia noturna familiar do lobo frontal; EAI = Epilepsia ausência da infância; SD = Síndrome de Dravet; HIV = Vírus da imunodeficiência humana; NMDA = N-metil-D-aspartato.

Fonte: Adaptado de Fisher et al, $2017^{2}$. 


\section{Exames complementares e diagnóstico}

A investigação complementar é conduzida associando-se a clínica à eletroencefalografia (EEG) e aos exames de imagem. O EEG é um exame não invasivo, auxiliar na classificação da crise (e escolha do melhor tratamento), na determinação do seu foco de origem, na identificação da síndrome epiléptica e no controle do tratamento. A sensibilidade de um único exame de EEG é inferior a $50 \%$, entretanto pode aumentar para $92 \%$ no quarto exame. A especificidade do exame, por sua vez, é superior a 90\% Desse modo, um EEG normal não exclui o diagnóstico de epilepsia, mas um exame positivo é altamente sugestivo deste diagnóstico ${ }^{11}$.

Já os exames de neuroimagem (ressonância magnética - RM e tomografia computadorizada - TC) são especialmente importantes na detecção de etiologias estruturais. Diante desta suspeita, são úteis tanto para a identificação do foco epileptogênico, quanto para o diagnóstico diferencial, como neoplasias ou lesões isquêmicas cerebrais. A RM apresenta sensibilidade superior à TC para a avaliação da epilepsia, contudo para o afastamento de causas emergenciais, como trauma, a TC é preferencial, detectando anormalidades estruturais em 34-56\% dos pacientes ${ }^{12}$. A RM do encéfalo é fundamental na investigação e manejo dos pacientes farmacorresistentes, sobretudo nos indivíduos com suspeita de etiologia estrutural passível de tratamento neurocirúrgico ${ }^{6,9}$.

Vale ressaltar que os exames complementares não são obrigatórios para o início do tratamento, o qual pode ser conduzido baseando-se exclusivamente na clínica.

As síndromes epilépticas constituem o agrupamento das epilepsias conforme características clínicas, eletroencefalográficas e de neuroimagem em comuns. Estas apresentam uma idade de acometimento, padrão de crises, e prognóstico típicos. Além disso, as síndromes frequentemente, apresentam comorbidades neuropsiquiátricas associadas, as quais demandam tratamento específicos e, portanto, devem ser diagnosticadas de forma precoce ${ }^{12}$.

\section{Abordagem terapêutica inicial da pessoa com epilepsia}

Após a primeira crise, pacientes sem fatores de risco para recorrência (como lesões estruturais nos exames de imagem ou achados epileptiformes no EEG) apresentam um risco de $25 \%$ de novo evento em dois anos. O tratamento instituído imediatamente após a primeira crise reduz a recorrência nos primeiros dois anos em $35 \%$, contudo, não implica em remissão sustentada de crises após 3-5 anos ${ }^{13}$.

Frente a isso, a decisão de quando iniciar o tratamento deve ser individualizada. Deve-se pesar os efeitos adversos da medicação, a frequência das crises e seu impacto na qualidade de vida do PCE, o risco de trauma ou morte súbita, bem como as indicações e contraindicações especificas de cada paciente. Caso os riscos excedam os benefícios, recomenda-se aguardar a ocorrência de uma segunda crise antes de iniciar o uso dos medicamentos ${ }^{6}$.

Em casos em que o diagnóstico de epilepsia está consolidado, os benefícios do tratamento são inquestionáveis, reduzindo a recorrência de crises em $57 \%$ em um ano e $73 \%$ em $4 \operatorname{anos}^{13,14}$. Na abordagem inicial, a monoterapia é ideal por ser mais segura e implicar em menos efeitos adversos. Sabe-se que quase metade dos pacientes com epilepsia recém diagnosticada alcançam remissão após a instituição da primeira monoterapia e não exigem substituição ou adição de outros medicamentos. Como regra geral, os medicamentos antiepilépticos de amplo espectro (como valproato, lamotrigina, levetiracetam e topiramato) são escolhas iniciais seguras para adultos com crises focais $\mathrm{e}$ generalizadas. Carbamazepina e fenitoína, contudo, têm indicação precisa para crises focais e podem exacerbar alguns tipos de crises generalizadas ${ }^{15}$.

O Quadro 5 apresenta um resumo das indicações dos medicamentos antiepilépticos e o Quadro 6 resume as propriedades farmacológicas dos fármacos disponíveis na APS. 
Quadro 5. Recomendações com nível de evidência para o tratamento da epilepsia em monoterapia.

\begin{tabular}{|c|c|}
\hline Cenário & Recomendação com níveis de evidência \\
\hline Adultos com crises de início focal & $\begin{array}{l}\text { Carbamazepina }{ }^{\mathrm{A}} \text {; Fenobarbital }{ }^{\mathrm{A}} \text {; Levetiracetam } \\
\text { Valproato }^{\mathrm{B}}\end{array}$ \\
\hline Crianças com crises de início focal & Oxcarbazepina ${ }^{A}$ \\
\hline Idosos com crises de início focal & $\begin{array}{l}\text { Gabapentina }^{\mathrm{A}} \text {; Lamotrigina } \\
\text { Topiramato }^{\mathrm{D} ;} \text { Valproato }^{\mathrm{D}} \text {. }\end{array}$ \\
\hline Adultos com crises tônico-clônicas generalizadas & $\begin{array}{l}\text { Valproato }^{\mathrm{c}} \text {; Fenobarbital'; Fenitoína }{ }^{\mathrm{c}} \text {; } \\
\text { Carbamazepina }^{\mathrm{c}} \text {; Topiramato } ; \text { Lamotrigina }^{\mathrm{c}} \text {; } \\
\text { Oxcarbazepina }^{\mathrm{C}} \text {; Gabapentina }{ }^{\mathrm{D}} \text {; Levetiracetam }\end{array}$ \\
\hline Crianças com crises tônico-clônicas generalizadas & $\begin{array}{l}\text { Valproato }^{\mathrm{c}} \text {; Fenobarbital'; Fenitoína } \\
\text { Carbamazepina }^{\mathrm{c}} \text {; Topiramato }\end{array}$ \\
\hline Crianças com crises de ausência & Valproato $^{\wedge}$, Etossuximida ${ }^{\wedge}$ Lamotrigina ${ }^{\mathrm{C}}$ \\
\hline
\end{tabular}

A - Nível de evidência A = Nível máximo de evidência científica proporcionada por estudos classe I (ensaios clínicos randomizados, controlados e duplo-cegos e/ou metanálises com duração de pelo menos 1 ano) - medicamentos eficazes e bem estabelecidos como monoterapia;

B - Nível de evidência B = Medicamentos provavelmente eficazes como monoterapia avaliados por estudos classe II (ensaios clínicos randomizados < 1 ano de duração);

C - Nível de evidência C = Medicamentos possivelmente eficazes como monoterapia avaliados por estudos classe III (estudos abertos);

D - Nível de evidência D = Medicamentos potencialmente eficazes como monoterapia avaliados por estudos classe IV (relatos de caso ou opinião de especialistas);

Fonte: Adaptado de Glauser et al, $2013^{15}$.

Recomenda-se reavaliações com intervalo máximo de 3 meses para acompanhamento da adesão, eficácia do tratamento, efeitos colaterais e reações idiossincráticas. Pacientes estáveis devem ser acompanhados na APS, idealmente com duas a três consultas anuais ${ }^{6}$. Considera-se remissão da epilepsia a ausência de sintomas por período mínimo de 10 anos, quando em tratamento, e de 5 anos para aqueles sem terapia medicamentosa ${ }^{2}$.

A decisão de quando interromper o tratamento ainda não é consenso pois a chance de recorrência após a retirada de um medicamento que promoveu controle por 2 anos varia entre 12-66\% ${ }^{17}$. Logo, deve-se individualizar a decisão, considerando-se manter $o$ medicamento caso haja fatores de risco para a recorrência da doença. Para pacientes com bom controle sintomático há mais de 2-5 anos pode-se avaliar a retirada das medicaçõe ${ }^{6,9}$.
Além do tratamento das crises, a abordagem psicossocial é fundamental, pois a epilepsia traz estigma social, limitações de atividades habituais, comprometimento de humor, de relacionamentos interpessoais e do trabalho. Estima-se que mais da metade das pessoas com epilepsias de difícil controle apresentem distúrbios psiquiátricos associados ${ }^{18}$. Logo, é imprescindível orientar quanto ao caráter crônico da condição, ressaltando-se que o tratamento com boa adesão tem o potencial de promover o controle dos sintomas e melhorar a qualidade de vida.

$\mathrm{O}$ incentivo ao suporte familiar, especialmente para motivar a adesão ao tratamento, também é fundamental. Com uma rede de apoio estruturada, o indivíduo torna-se mais resiliente o que impacta em seu bem-estar ${ }^{18}$. O Quadro 7 elenca as principais orientações de estilo de vida que devem ser frisadas ao PCE e sua família. 
Quadro 6. Propriedades dos principais medicamentos disponíveis na Atenção Primária à Saúde.

\begin{tabular}{|c|c|c|c|c|}
\hline Medicamento & Fenitoína & Fenobarbital & Carbamazepina & $\begin{array}{l}\text { Valproato de } \\
\text { sódio }\end{array}$ \\
\hline Apresentação & $\begin{array}{ll}\text { - } & 100 \mathrm{mg}-\mathrm{cp} \\
\text { - } & 20 \mathrm{mg} / \mathrm{ml} \text { - } \\
& \text { suspensão oral } \\
\text { - } & 50 \mathrm{mg} / \mathrm{ml} \text { - } \\
& \text { solução } \\
& \text { injetável }\end{array}$ & $\begin{array}{ll}\text { - } & 100 \mathrm{mg}-\mathrm{cp} \\
\text { - } & 40 \mathrm{mg} / \mathrm{ml} \text { - } \\
& \text { suspensão oral } \\
\text { - } & 100 \mathrm{mg} / \mathrm{ml} \text { - } \\
& \text { solução } \\
& \text { injetável }\end{array}$ & $\begin{array}{ll}\text { - } & 200 \mathrm{mg}-\mathrm{cp} \\
\text { - } & 400 \mathrm{mg}-\mathrm{cp} . \\
\text { - } & 20 \mathrm{mg} / \mathrm{ml}- \\
& \text { suspensão oral }\end{array}$ & 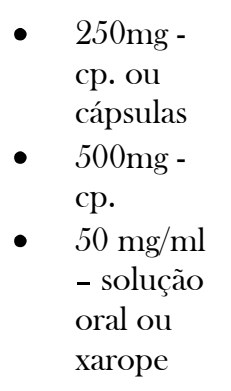 \\
\hline Dose inicial & $1 \mathrm{cp} 2 \mathrm{x} / \mathrm{dia}$ & $1 c p 1 x /$ dia & $1 \mathrm{cp} \mathrm{2x/dia}$ & $1 \mathrm{cp} \mathrm{2x/dia}$ \\
\hline $\begin{array}{l}\text { Manutenção (níveis } \\
\text { terapêuticos) }\end{array}$ & $6-8 \mathrm{mg} / \mathrm{kg} /$ dia & $1-2,5 \mathrm{mg} / \mathrm{kg} / \mathrm{dia}$ & $600-1000 \mathrm{mg} / \mathrm{dia}$ & $\begin{array}{l}1000- \\
2000 \mathrm{mg} / \mathrm{dia}\end{array}$ \\
\hline Contraindicação & $\begin{array}{l}\text { Crises de ausência } \\
\text { e crises } \\
\text { mioclônicas }\end{array}$ & & $\begin{array}{l}\text { Crises mioclônicas, de } \\
\text { ausência ou atônicas e } \\
\text { gestantes }\end{array}$ & $\begin{array}{l}\text { Gestantes e } \\
\text { crianças } \\
\text { menores de } 2 \\
\text { anos }\end{array}$ \\
\hline Efeitos adversos & $\begin{array}{l}\text { Sonolência, ataxia, } \\
\text { disartria, nistagmo, } \\
\text { diplopia, acne, } \\
\text { hirsutismo, anemia, } \\
\text { hiperplasia gengival }\end{array}$ & $\begin{array}{l}\text { Sedação, tonturas, } \\
\text { cefaleia, alterações } \\
\text { de humor, redução } \\
\text { da concentração, } \\
\text { lentificação } \\
\text { psicomotora }\end{array}$ & $\begin{array}{l}\text { Náuseas, cefaleia, } \\
\text { tonturas, sedação, } \\
\text { fadiga, diplopia, } \\
\text { nistagmo, } \\
\text { incoordenação motora, } \\
\text { ganho ponderal e } \\
\text { redução da densidade } \\
\text { óssea. }\end{array}$ & $\begin{array}{l}\text { Náuseas, } \\
\text { dispepsia, } \\
\text { ganho } \\
\text { ponderal, } \\
\text { sonolência, } \\
\text { edema, } \\
\text { alopécia, } \\
\text { síndrome dos } \\
\text { ovários } \\
\text { policísticos, } \\
\text { plaquetopenia, } \\
\text { parkinsonismo, } \\
\text { gota, demência }\end{array}$ \\
\hline $\begin{array}{l}\text { Interação } \\
\text { medicamentosa }\end{array}$ & $\begin{array}{l}\text { Amiodarona, } \\
\text { fluoxetina, } \\
\text { isoniazida, } \\
\text { antifúngicos } \\
\text { azólicos e valproato } \\
\text { de sódio }\end{array}$ & & $\begin{array}{l}\text { Inibidores da CYP3A4, } \\
\text { como macrolídeos, } \\
\text { fluoxetina e suco de } \\
\text { grapefruit }\end{array}$ & $\begin{array}{l}\text { Elevação sérica } \\
\text { quando } \\
\text { coadministrado } \\
\text { com fenitoína }\end{array}$ \\
\hline Idiossincrasias & $\begin{array}{l}\text { Rash, SSJ, NET, } \\
\text { reações de } \\
\text { hipersensibilidade }\end{array}$ & & $\begin{array}{l}\text { SSJ, NET, lúpus } \\
\text { induzido por drogas, } \\
\text { hepatotoxicidade, } \\
\text { hipersensibilidade }\end{array}$ & $\begin{array}{l}\text { Hepatotoxicida } \\
\text { de, pancreatite } \\
\text { aguda }\end{array}$ \\
\hline
\end{tabular}

Siglas: $\mathrm{cp}=$ comprimido; SSJ = Síndrome de Stevens-Johnson; NET = Necrólise epidérmica tóxica.

Fonte: Adaptado de Abou-Khalil, $2016^{16}$. 
Quadro 7. Orientações psicossociais para pessoas com epilepsia e seus familiares.

\begin{tabular}{|l|}
\hline $\begin{array}{l}\text { Aspectos emocionais: Orientar benefício da psicoterapia para o processo adaptativo de doença e indagar ativamente } \\
\text { sobre o bem-estar do paciente a cada consulta. }\end{array}$ \\
\hline $\begin{array}{l}\text { Controle de crises: O paciente deve ser incentivado a registrar os fatores desencadeantes de suas crises em um } \\
\text { diário, para que possa evitá-los. }\end{array}$ \\
\hline $\begin{array}{l}\text { Sono: A privação de sono é um dos principais estímulos ao desencadeamento de crises. Os pacientes devem dormir } \\
\text { um número mínimo de seis a oito horas por noite e evitar o uso de cafeína no período noturno. }\end{array}$ \\
\hline $\begin{array}{l}\text { Luzes brilhantes, fluorescentes ou que pisquem: Alguns pacientes podem ter crises desencadeadas por estímulos } \\
\text { luminosos. Orientar evitar locais como clubes e festas, reduzir a luz do computador e celular e utilizar óculos escuros } \\
\text { em ambientes muito luminosos. }\end{array}$ \\
\hline $\begin{array}{l}\text { Direção de veículos: Pessoas com epilepsia podem dirigir desde que estejam livres de crises por um período } \\
\text { superior a } 12 \text { meses e apresentem um parecer do médico assistente. }\end{array}$ \\
\hline $\begin{array}{l}\text { Consumo de álcool e abuso de drogas ilícitas: Ambos os comportamentos podem precipitar ou agravar crises pré- } \\
\text { existentes, portanto devem ser contraindicados ativamente pela equipe de saúde. }\end{array}$ \\
\hline $\begin{array}{l}\text { Intercorrências de saúde ou traumas: Podem exacerbar a ocorrência de crises. O paciente deve ser orientado a } \\
\text { informar aos profissionais de saúde sobre sua epilepsia e sobre os medicamentos de que faz uso. }\end{array}$ \\
\hline
\end{tabular}

Fonte: Adaptado de Mula et al, $2016^{18}$.

\section{Quando encaminhar o paciente com epilepsia?}

Segundo a $17^{\mathrm{a}}$ Portaria Conjunta do Ministério da Saúde ${ }^{6}$, os atendimentos na rede podem ocorrer nos níveis primário, secundário e terciário de atenção. Os clínicos gerais, pediatras e médicos de família e comunidade podem controlar cerca de $50 \%$ das crises epilépticas com medicamentos disponíveis na APS em monoterapia. Neurologistas e neurologistas pediátricos podem proporcionar controle de crises a adicionais $20 \%$ dos PCE com medicamentos disponíveis na Relação Nacional de Medicamentos Essenciais (RENAME) em monoterapia ou politerapia. Por fim, epileptologistas, neurocirurgiões e equipes especializadas em epilepsia podem proporcionar controle a cerca de $50 \%$ dos pacientes com epilepsia farmacorresistente ${ }^{19}$.

Desse modo, é importante que esse referenciamento seja feito de forma adequada. $\mathrm{O}$ cuidado de pacientes com epilepsia que apresentam bom controle, ausência de comorbidades e boa tolerância medicamentosa deve ser realizado na APS. Entretanto, em algumas situações há necessidade de encaminhamento para o especialista focal ou para serviços de urgência e emergência. O quadro 8 elenca os principais sinais de alerta para o encaminhamento para a atenção secundária e o quadro 9 cita indicações de encaminhamento para serviços de urgência e emergência.

\section{Quadro 8. Sinais de alarme para o encaminhamento para o especialista focal (neurologista).}

\begin{tabular}{|l|}
\hline Múltiplas crises e de difícil controle \\
\hline Suspeita de doença sistêmica ou neurológica aguda \\
\hline Extremos de idade \\
\hline Histórico de estado de mal epiléptico prévio \\
\hline Síndrome epiléptica grave \\
\hline Esquemas terapêuticos complexos e com múltiplas interações medicamentosas \\
\hline Comprometimento cognitivo e de desenvolvimento cerebral \\
\hline $\begin{array}{l}\text { Pacientes com lesões neurológicas estruturais nos exames de imagem para avaliação de indicação de tratamento } \\
\text { neurocirúrgico }\end{array}$ \\
\hline
\end{tabular}

Fonte: Adaptado de Engel et al, $2016^{20}$. 
Quadro 9. Sinais de alarme para encaminhamento para serviço de urgência e emergência.

\begin{tabular}{|l|}
\hline Alteração do exame neurológico no período interictal \\
\hline Alteração persistente de nível de consciência entre as crises \\
\hline Suspeita de crise sintomática aguda \\
\hline Crises com duração superior a 5 minutos ou crises reentrantes sem recuperação de consciência entre elas \\
\hline Trauma associado às crises \\
\hline Descompensação aguda de comorbidade clínica ou psiquiátrica \\
\hline
\end{tabular}

Fonte: Adaptado de Iyer et al, 2012.

Para fins ilustrativos, o fluxograma 1 resume os pontos essenciais sobre a abordagem da pessoa com epilepsia na APS.

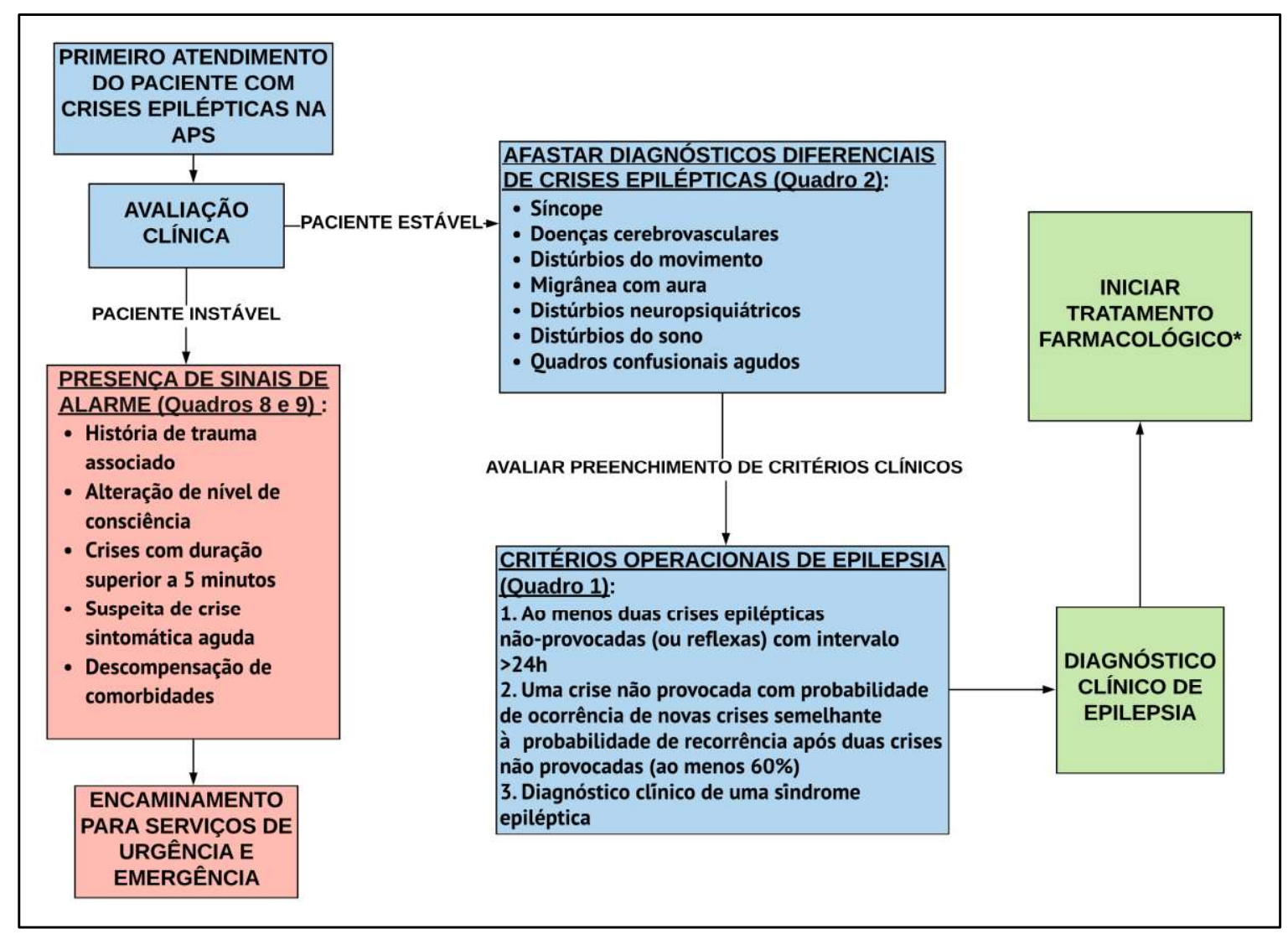

Fluxograma 1. Abordagem inicial da pessoa com crises epilépticas na atenção primária à saúde. * Deve-se iniciar o tratamento com um único medicamento antiepiléptico em monoterapia, com a menor dose eficaz, em titulação lenta, até o controle total das crises epilépticas ou o surgimento de efeitos adversos intoleráveis. Caso haja falha da primeira monoterapia, deve-se sempre tentar fazer a substituição gradual por uma nova monoterapia em sequência, seguindo os mesmos princípios de tratamento. Sigla: APS = atenção primária à saúde. Fonte: Adaptado de Fisher et al, 20141; PCDT, 2018 (Protocolo Clínico e Diretrizes Terapêuticas da Portaria Conjunta No 17 de 21 de junho de 2018)6; Iyer et al, 201221. 


\section{Epilepsia farmacorresistente}

Epilepsia farmacorresistente é aquela na qual ocorre falha na remissão de crises apesar de uma intervenção adequada e bem tolerada com, ao menos, dois medicamentos antiepiléticos em monoterapia ou politerapia, o que pode ocorrer em cerca de $15 \%$ dos pacientes com epilepsia focal ${ }^{19}$. É um dos principais desafios do tratamento da epilepsia na APS.

Os pacientes que não responderem ao tratamento otimizado com os medicamentos disponíveis na rede básica têm indicação de referenciamento para profissionais especialistas em Neurologia ${ }^{6}$, no entanto, primeiramente é necessário confirmar se há boa adesão ao tratamento e se o fármaco foi corretamente indicado. Pacientes pouco aderentes ou fazendo uso de medicamentos inadequados não podem ser considerados farmacorresistentes, até que a terapêutica seja corrigida.

Também é importante a revisão do diagnóstico, pois outras condições clínicas podem cursar com crises não-epilépticas. Alguns PCE previamente responsivas ao tratamento podem tornar-se refratárias por apresentarem crises cuja causa é distinta de sua epilepsia, chamadas de crises sintomáticas agudas (Quadro 10) ${ }^{22}$. Estas crises têm correlação temporal com insultos cerebrais ou sistêmicos agudos e não tendem a recorrer após tratamento do insulto precipitante.

Quadro 10. Principais causas de crises sintomáticas agudas.

\begin{tabular}{|c|c|}
\hline Etiologia & Principais condições determinantes \\
\hline Metabólica & $\begin{array}{l}\text { Hipoglicemia; } \text { Cetoacidose }^{2} \text {; Hiponatremia }{ }^{3} \text {; Hipocalcemia }{ }^{4} \text {; Hipomagnesemia; } \\
\text { Hiperuricemia; Elevação de creatinina }\end{array}$ \\
\hline Estrutural & Acidente vascular encefálico; trauma crânio-encefálico \\
\hline Exógena & Libação ou abstinência alcoólica; Overdose ${ }^{8}$ Intoxicação exógena aguda ${ }^{9}$ \\
\hline Infecciosa & Meningites, Encefalites \\
\hline
\end{tabular}

1 - HGT < 36mg/dl; 2 - HGT > 450mg/dl; 3 - Sódio < 115mg/dl; 4 - Cálcio < 5mg/dl; 5 - Magnésio < 0,8mg/dl; 7 - Creatinina > $19 \mathrm{mg} / \mathrm{dl} ; 8$ - Overdose de drogas excitotóxicas (principalmente crack e cocaína); 9 - Principais drogas: benzodiazepínicos, meperidina, anfetaminas, antidepressivos tricíclicos, lítio. Fonte: Adaptado de Beleza et al, $2012^{22}$.

Em vista da importância do cuidado próximo do PCE (especialmente se não houver controle adequado das crises) o Fluxograma 2 resume algumas das principais condutas norteadoras do acompanhamento ambulatorial destes pacientes. 


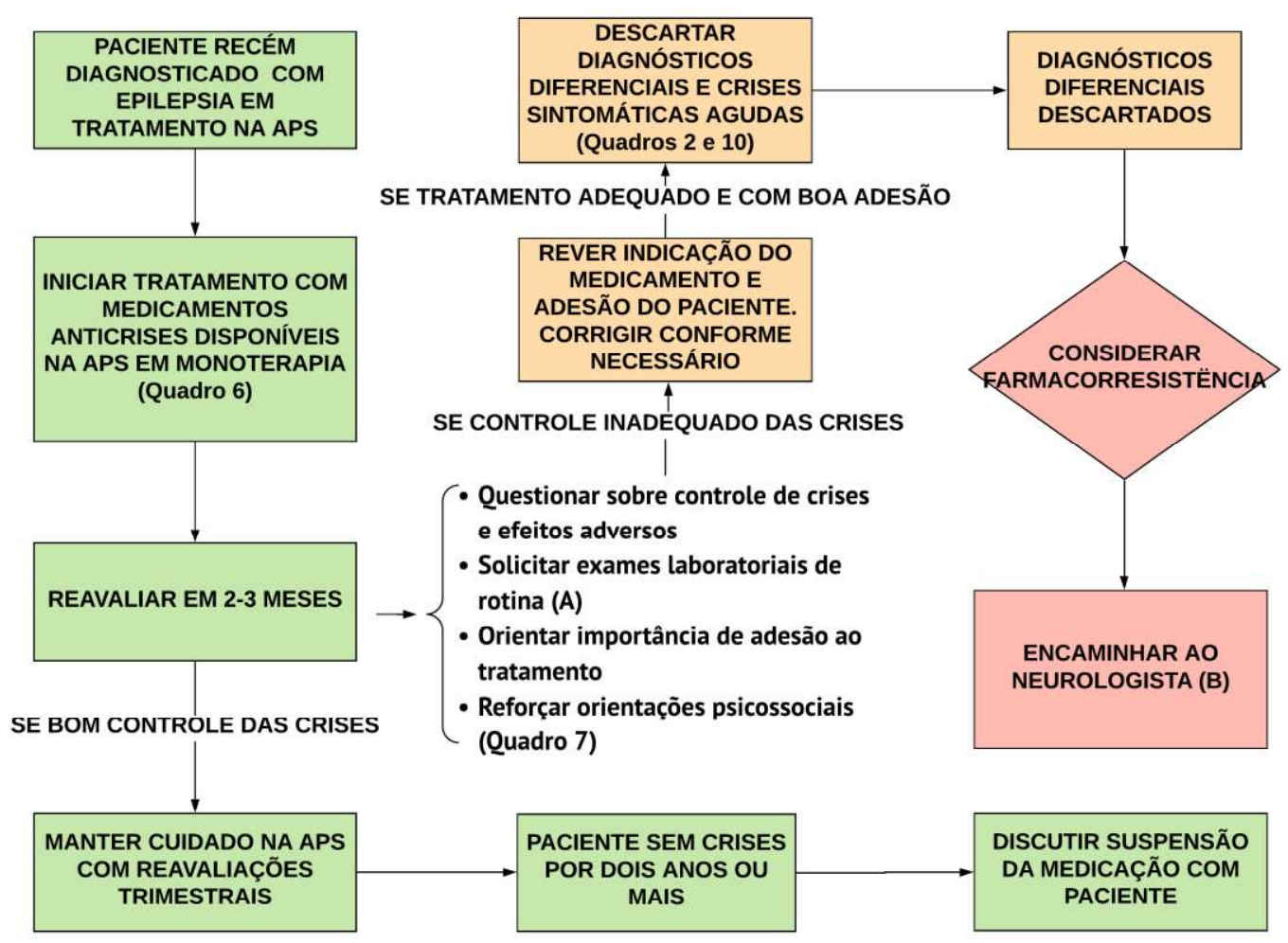

Fluxograma 2. Orientações gerais para o tratamento e acompanhamento da pessoa com epilepsia na atenção primária à saúde. A) Recomenda-se a realização anual dos seguintes exames laboratoriais: hemograma, provas de função hepática (ALT, AST, gama-GT, fosfatase alcalina), eletrólitos (sódio e potássio), perfil lipídico (colesterol total e frações, triglicerídeos), função tireoidiana (TSH e T4 livre).

B) Após falha na segunda tentativa de monoterapia, pode-se tentar a combinação de dois fármacos antiepilépticos e encaminhar o paciente a centros especializados no tratamento de epilepsias, aptos a oferecer tratamentos nãomedicamentosos, como tratamento neurocirúrgico, estimulação vagal, dieta cetogênica, medicamentos experimentais em pesquisa clínica. Sigla: APS = Atenção Primária à Saúde. Fonte: Adaptado de PCDT, 2018 (Protocolo Clínico e Diretrizes Terapêuticas da Portaria Conjunta No 17 de 21 de junho de 2018)6; Devinsky et al, 20189.

\section{O paciente com epilepsia e a pandemia da COVID-19}

A Organização Mundial da Saúde decretou oficialmente, em março de 2020, a pandemia da COVID-19 e, desde então, importantes restrições foram impostas a indivíduos de todo o globo. Em relação à atenção à saúde, o atendimento de rotina, exames e estudos clínicos foram suspensos, e muitas doenças crônicas como a epilepsia deixaram de ser adequadamente tratadas, salvo apenas nos casos em que possibitou-se o uso da telemedicina (vídeo, telefone, whatsapp, etc.).

À medida que o conhecimento sobre a COVID19 foi aumentando e adaptações se fizeram necessárias, muitas dúvidas iniciais dos PCE e seus cuidadores puderam ser gradativamente esclarecidas.

Pacientes com epilepsia e COVID-19 - o que já se sabe e quais são as recomendações?

1. O vírus da COVID-19 não é causa direta de epilepsia. Crises epilépticas e outras manifestações neurológicas em vigência de formas graves da COVID-19 parecem ser secundárias à injúria cerebral aguda pelo vírus, levando à encefalite, encefalopatia hipóxicoisquêmica, e/ou doença encefalovascular isquêmica ou hemorrágica;

2. Pacientes com epilepsia não sofrem risco aumentado de infecção pelo vírus da COVID19 , nem parecem ter maior probabilidade de adquirir a forma grave da doença;

3. Diversos medicamentos em uso experimental para o tratamento da COVID-19 (hidroxicloroquina, azitromicina, antivirais, imunoglobulina, etc.) não possuem contraindicações formais para uso em PCE. O riscobenefício deve ser, portanto, avaliado individualmente e amplamente discutido entre o médico e seu paciente;

4. O PCE deve manter hábitos de vida saudáveis, apesar das medidas de isolamento social, procurando ter sono regular, alimentação saudável, e atividade física regular, medidas fundamentais também para manutenção da sua saúde mental; 
5. Evitar consumo de bebidas alcoólicas, drogas lícitas (sem acompanhamento médico) ou ilícitas;

6. Procurar garantir acesso ao seu médico de confiança, seja através da telemedicina ou outras formas de consulta não presenciais, minimizando o risco de exposição e contágio da COVID-19;

7. Garantir o seu suprimento de medicamentos antiepilépticos com antecedência, por 3-6 meses, sempre que possível;

8. Ter sempre à mão medicamentos de resgate para crises epilépticas agudizadas, como benzodiazepínicos de ação rápida, para uso quando necessário;

9. Adesão ao tratamento e evitar comportamentos e fatores precipitantes de crises epilépticas, evitando descompensação das crises epilépticas e necessidade de atendimento emergencial de saúde em instituições já sobrecarregadas pelo atendimento aos casos de COVID-19. Porém, em caso de crises epilépticas com mais de 5 minutos de duração, ou salva de crises epilépticas sem recuperação da consciência entre elas, o PCE deve ser levado imediatamente ao hospital ${ }^{7,823}$.

\section{Conclusão}

As epilepsias acarretam grande comprometimento da qualidade de vida quando tratadas inadequadamente. O manejo da maioria dos PCE pode ser conduzido preferencialmente na APS, foco da articulação do cuidado, atenção longitudinal e integral à saúde. Apenas os pacientes com sinais de alerta e aqueles com epilepsias farmacorresistentes necessitarão ser encaminhados para o especialista.

Entretanto, não-especialistas ainda não se sentem plenamente seguros na atenção destes pacientes, haja vista a complexidade de apresentações clínicas das epilepsias, a multiplicidade de interações medicamentosas a que estes pacientes estão sujeitos; ao desafio do cuidado das comorbidades neuropsiquiátricas associadas e a dificuldade no controle das crises dos pacientes com resistência farmacológica.

Assim sendo, é imprescindível que haja discussões por parte dos MFC sobre o diagnóstico de epilepsia, sobre as terapias medicamentosas disponíveis no SUS e, também, sobre quais pessoas devem ser referenciadas para outros pontos de atenção da rede. Sob este prisma, uma revisão que traga essas informações de forma concisa e de fácil acesso representa válida contribuição à APS, no entanto, de forma complementar, ressalta-se que uma proposta de educação continuada acerca deste tópico em trabalhos futuros (abordando, por exemplo, as particularidades de manejo de outros grupos de atenção, como crianças, idosos ou gestantes) é fundamental.

\section{Reconhecimento}

A autora KL possui Bolsa de Pesquisa PQ2 do CNPq (Conselho Brasileiro de Desenvolvimento Científico e Tecnológico, Brasil) (Processo no 304936 / 2017-0) e recebeu apoio financeiro da FAPESC / CNPq -PRONEM - $n^{\circ}$ 2020 TR736.

\section{Referências}

1. Fisher RS, Acevedo C, Arzimanoglou A, Bogacz A, Cross JH, Elger CE, et al. ILAE official report: a practical clinical definition of epilepsy. Epilepsia. 2014 Apr;55(4):475-82.

https://doi.org/10.1111/epi.12550

2. Fisher RS, Cross JH, French JA, Higurashi N, Hirsch E, Jansen FE, et al. Operational classification of seizure types by the International League Against Epilepsy: position paper of the ILAE Commission for Classification and Terminology. Epilepsia. 2017 Mar;58(4):522-30. https://doi.org/10.1111/epi.13670

3. Fiest KM, Sauro KM, Wiebe S, Patten SB, Kwon CS, Dykeman J, et al. Prevalence and incidence of epilepsy: a systematic review and meta-analysis of international studies. Neurology. 2017

Jan;88(3):296-303.

https://doi.org/10.1212/WNL.0000000000003509

4. Brasil. Ministério da Saúde. Secretaria de Atenção à Saúde. Departamento de Atenção Básica.

Acolhimento à demanda espontânea: queixas mais comuns na Atenção Básica / Ministério da Saúde, Secretaria de Atenção à Saúde, Departamento de Atenção Básica. - 1. ed.; 1. reimp. - Brasília: Ministério da Saúde, 2013.290 p. nil. - (Cadernos de Atenção Básica n. 28, Volume II) - Página 39. https:/bvsms.saude.gov.br/bvs/publicacoes/acolhime nto demanda espontanea queixas comuns cab28v 2.pdf

5. Epilepsy: a public health imperative. Summary. Geneva: World Health Organization; 2019 (WHO/MSD/MER/19.2)

https://www.who.int/mental_health/neurology/epilep sy/report_2019/en/

6. Ministério da Saúde. Portaria conjunta N. 17, de 21 de junho de 2018. Protocolo clínico e diretrizes terapêuticas - Epilepsia. Acessado em

30/novembro/2020. https://www.in.gov.br/materia/Lasset_publisher/Kujrw0TZC2Mb/content/id/27391 635/do1-2018-06-27-portaria-conjunta-n-17-de-21de-junho-de-2018-27391620

7. Volkers N. Will the pandemic give a permanent 
boost to telemedicine for epilepsy? Epigraph [periódico na Internet]. 2020 [acesso em $2020 \mathrm{Dez}$ 01];22(4):1-10. Disponível em:

https://www.ilae.org/iournals/epigraph/epigraph-vol22-issue-4-summer-2020/will-the-pandemic-give-apermanent-boost-to-telemedicine-for-epilepsy

8. French JA, Brodie MJ, Caraballo R, Devinsky O, Ding D, Jehi L, et al. Keeping people with epilepsy safe during the COVID-19 pandemic. Neurology. 2020 Jun;94(23):1032-7.

https://doi.org/10.1212/WNL.0000000000009632

9. Devinsky O, Vezzani A, O'Brien TJ, Jette N, Scheffer IE, Curtis M, et al. Epilepsy. Nat Rev Dis Primers. 2018 May;4(1):18024. https://doi.org/10.1038/nrdp.2018.24

10. Xu Y, Nguyen D, Mohamed A, Carcel C, Li Q, Kutlubaev MA, et al. Frequency of a false positive diagnosis of epilepsy: A systematic review of observational studies. Seizure. 2016 Oct;41(1):167 74. https://doi.org/10.1016/j.seizure.2016.08.005

11. Kural MA, Duez L, Hansen VS, et al. Criteria for defining interictal epileptiform discharges in EEG: a clinical validation study. Neurology. 2020 May;94(20):e2139-47. https://doi.org/10.1212/WNL.0000000000009439

12. Scheffer I, Berkovic S, Capovilla G, et al. ILAE classification of the epilepsies: position paper of the ILAE Commission for Classification and Terminology. Epilepsia. 2017 Apr;58(4):512-21. https://doi.org/10.1111/epi.13709

13. Krumholz A, Wiebe S, Gronseth GS, et al. Evidence-based guideline: management of an unprovoked first seizure in adults - report of the Guideline Development Subcommittee of the American Academy of Neurology and the American Epilepsy Society. Neurology. 2015 Apr;84(16):170513. https://doi.org/10.1212/WNL.0000000000001487

14. Kwan P, Brodie MJ. Early identification of refractory epilepsy. New England Journal of Medicine. 2000 Feb;342:314-9. https://www.nejm.org/doi/full/10.1056/nejm2000020 33420503

15. Glauser T, Ben-Menachem E, Bourgeois B, Cnaan A, Guerreiro C, Kalviainen R, et al. Updated ILAE evidence review of antiepileptic drug efficacy and effectiveness as initial monotherapy for epileptic seizures and syndromes. Epilepsia. 2013

Mar;54(3):551-63. https://doi.org/10.1111/epi.12074

16. Abou-Khalil BW. Antiepileptic drugs. Continuum (Minneap Minn). 2016 Feb;22(1):132-56. https://journals.lww.com/continuum/Abstract/2016/0 2000/Antiepileptic Drugs.12.aspx

17. Rizvi S, Ladino LD, Hernandez-Ronquillo L, Téllez-Zenteno JF. Epidemiology of early stages of epilepsy: risk of seizure recurrence after a first seizure. Seizure. 2017 Jul;49(1):46-53. https://doi.org/10.1016/j.seizure.2017.02.006

18. Mula M, Sander JW. Psychosocial aspects of epilepsy: a wider approach. BJPsych Open. 2016 Jul;2(4):270-4. https://doi.org/10.1192/bjpo.bp.115.002345

19. Kwan P, Arzimanoglou A, Berg AT, Brodie MJ, Allen Hauser W, Mathern G, et al. Definition of drug resistant epilepsy: consensus proposal by the ad hoc Task Force of the ILAE Commission on Therapeutic Strategies. Epilepsia. 2009 Nov;51(6):1069-77. https://doi.org/10.1111/j.1528$\underline{1167.2009 .02397 . \mathrm{x}}$

20. Engel Jr J. What can we do for people with drugresistant epilepsy? Neurology. 2016 Dec;87 (23):2483-9. https://doi.org/10.1212/WNL.00000000000034.07

21. Iyer PM, McNamara PH, Fitzgerald M, Smyth L, Dardis C, Jawad T, et al. A seizure care pathway in the emergency department: preliminary quality and safety improvements. Epilepsy Res Treat. 2012 May;273175.

https://www.hindawi.com/archive/2012/273175/

22. Beleza P. Acute symptomatic seizures: a clinically oriented review. Neurologist. 2012 May;18(3):10919.

https://doi.org/10.1097/NRL.0b013e318251e6c3

23. Lavin B, Dormond C, Scantlebury MH, Frouin PY, Brodie MJ. Bridging the healthcare gap: building the case for epilepsy virtual clinics in the current healthcare environment. Epilepsy Behav. 2020 Jul;111:107262. https://doi.org/10.1016/j.yebeh.2020.107262 